\title{
Uplink Interference Management for Coexisting MIMO Femtocell and Macrocell Networks: An Interference Alignment Approach
}

\author{
Basak Guler, Student Member, IEEE, and Aylin Yener, Senior Member, IEEE
}

\begin{abstract}
This paper considers uplink interference management for two-tier cellular systems by way of Interference Alignment (IA). In order to manage the uplink interference caused by macrocell users at the femtocell base stations (FBS), cooperation between macrocell users with the closest femtocell base stations is proposed with the goal of aligning the received signals of macrocell users in the same subspace at multiple FBSs. The precoder design for macrocell users is accomplished using successive semidefinite programming relaxations. The proposed solution aims to minimize the cross-tier interference leaked to the femtocells while providing the macrocell users with a minimum received signal to interference plus noise ratio (SINR) at the macrocell base station (MBS). Intra-tier femtocell interference is dealt with minimum mean squared error (MMSE) interference suppression. Numerical results demonstrate that the proposed two-tier interference management approach improves the performance of femtocell users, while maintaining the desired quality of the communication channel of macrocell users.
\end{abstract}

Index Terms-Femtocells/small cells, two-tier networks, uplink interference management, interference alignment, MMSE interference suppression.

\section{INTRODUCTION}

$\mathbf{N}$ EXT generation wireless networks are expected to provide a diverse range of broadband services to meet subscriber demands. Femtocells are a promising direction to improve the performance for in-home users while reducing the load on the cellular (macrocell) network [1]. Femtocells require no infrastructure as they are plug-and-play devices that are connected to the internet backhaul [2]. A main deployment challange is that femtocells operate in the licenced band, and consequently have to share the radio resources and coexist with the cellular network. Solutions proposed to guarantee coexistence range from partitioning the frequency resources between the two networks with closed access, to allowing cellular (macrocell) users to be served by femtocell base stations with open access [1].

An alternative approach is to have users of a particular tier to be served by base stations of that tier, i.e., keeping the closed access, while sharing all frequency resources across

Manuscript received August 1, 2013; revised November 27, 2013; accepted January 10, 2014. The associate editor coordinating the review of this paper and approving it for publication was T. J. Lim.

An earlier version of this work was presented in part at the IEEE Global Communications Conference (GLOBECOM), December 2011.

The authors are with the Department of Electrical Engineering, The Pennsylvania State University, University Park, PA, 16802 USA (e-mail: basak@psu.edu; yener@ee.psu.edu).

Digital Object Identifier 10.1109/TWC.2014.030314.131408 tiers. In such an architecture, in order to ensure peaceful coexistence, effective management of cross-tier interference is of utmost importance. In the uplink, in particular, a macrocell user operating in the same band as femtocell users may cause unacceptably high interference levels, if it is close to the femtocell base station supporting the aforementioned femtocell users, and far away from its own macrocell base station. Additionally, the fact that femtocells can be deployed in an ad hoc fashion anywhere within a macrocell, and can be removed as easily, adds to the critical importance of interference management. Notwithstanding the importance of this issue, the concerns listed above renders jointly optimal design of the two networks impractical due to the complexity and overhead associated with a large dynamic network. Consequently, a computationally manageable yet effective interference management strategy is needed.

Interference management has been an important design element for multiuser systems in the past two decades. Judicious receiver design for interference limited systems, e.g., CDMA, and multiuser MIMO, proves useful for interference cancellation [3]. In addition to multiuser detection, transmit power control [4], and joint design of transmitters and receivers [5], [6] offer interference mitigation needed in interference limited systems. Power control has also been an important issue for co-existence of two-tier networks [7], [8]. While the aforementioned techniques have been designed primarily for multi-transmitter single receiver, i.e., multiple access systems, interference alignment has recently been proposed for multitransmitter multi-receiver models, i.e., interference networks.

Interference Alignment (IA) has been shown to achieve the degrees of freedom for the $K$-user interference channel [9] by aligning the interfering signals in a lower dimensional subspace at multiple receivers simultaneously. Perfect IA for multiantenna systems has only been achieved for networks with small number of users. Thus, for practical scenarios, i.e., when $K \geq 4$, distributed algorithms have been proposed to approximately align the interference while allowing some interference leakage [10]-[12]. These algorithms are developed for $K$-user interference channels, in which each transmitter has a distinct intended receiver, and the remaining transmitters are considered as interferers for that receiver. As an example, the algorithms proposed in [10] use channel reciprocity, and iterate between the receivers and transmitters at each step, by reversing the communication direction as in [13], in order to minimize the leaked interference/maximize the SINR of the 
intended signal, respectively. By contrast, our system model is a two-tier system that consists of many interfering multiple access channels.

Interference alignment for femtocell networks has recently been considered in settings different than ours, namely with orthogonal resource allocation. IA methods proposed for $K$ user interference channels have been used in [14] and [15] for mitigating the intra-tier femtocell interference in the downlink of a split-frequency femtocell-macrocell network, in which macrocell and femtocells are assigned separate frequency bands. The three user perfect interference alignment scheme has been utilized in [16] for managing the interference between picocells and a macrocell. An adaptive subband partitioning method is proposed in [14] to mitigate the femtocellto-femtocell interference, with each femtocell supporting a single user. In this sense, the network model becomes similar to a $K$-user interference channel, to which interference alignment techniques as proposed for the original single-tier network can be applied. Reference [15] considers the intratier (femtocell-to-femtocell) interference management problem in a downlink of a femtocell network, and studies the game-theoretic strategies for femtocells. In this reference, orthogonal resource allocation is done which enables treating the femtocells forming a cluster as a $K$-user interference channel. By contrast, our scheme considers the inter-tier uplink interference management problem in a femtocell-macrocell network, aligning the interfering signals of one tier at the receivers of the other tiers, and multiple simultaneous users are allowed in each femtocell and the ensuing two-tier interference alignment scheme. We note that while our approach does not involve explicit frequency partitioning between the tiers, i.e., relies solely on the space dimensions, allowing for greater flexibility, it is possible to have our scheme accompany a frequency partitioning scheme and increase the number of uplink users sharing each subband as well. Our methods assume cooperation amongst the femtocells within a cluster in a similar manner to cooperative multi-cell networks [17]. For a detailed discussion on the impact of joint-cell decoding on the underlying backhaul we refer to [18], [19], and to [20] for an application of IA to multi-cell joint decoding. The coordination between the macrocell users and FBSs within each cluster can be enabled by an access point that gathers the channel state information of the dominant macrocell users to design the macrocell user precoders and to notify each FBS in the cluster of its interference subspace. These access points may be realized in a similar fashion to the femtocell access points inherent in femtocell-macrocell networks.

In this paper, we take the viewpoint of managing the interference caused by the macrocell users (transmitters) to the uplinks of femtocells in their vicinity by aligning their signals at the right femtocell base stations (receivers). In order to manage the uplink interference caused by the macrocell users at the femtocell base stations (FBS), one can surmise using joint detection and interference cancellation. Given the potential complexity and overhead, however, this global approach can quickly become infeasible. We posit that a more viable approach to this two-tier interference management problem is by leveraging the coordination between a group of FBS and the macrocell users that are causing high interference to this group of FBSs. Specifically, using the principle of interference alignment (IA), we can align the received signals from these macrocell users in a lower dimensional subspace at multiple FBSs simultaneously, and use the remaining degrees of freedom to improve the detection performance of the femtocell users. While interference alignment helps the femtocell users to eliminate macrocell interference, this should not come at the expense of communication quality for the macrocell users. Our approach is to design the interference aligning precoders of macrocell users subject to individual SINR constraints at their MBS, thus making sure they can communicate reliably while minimizing their interference to the femtocells.

Toward accomplishing our goal, we propose to align the received macrocell interference as much as possible subject to minimum SIR constraints for each macrocell user (MU) at the FBSs, by employing successive semidefinite programming (SDP) relaxations. After interference alignment, a precodingdecoding scheme is used at the FBSs to minimize the sum MSE of the femtocell users (FU), which we call the coordinated MMSE approach. For comparison purposes, we also propose employing a zero-forcing constraint in the minimum sum MSE problem in order to eliminate the leftover aligned macrocell interference separately at each FBS, which we call the coordinated zero-forcing approach.

Numerical results demonstrate the benefits of the proposed IA algorithm, and that these benefits increase as the number of interfering macrocell users increase. The number of macrocell users that can be aligned simultaneously depends on the minimum SINR requirements at the MBS, more users can be aligned when the minimum SINR requirements are decreased. It is also observed that separately zero-forcing the leaked macrocell interference can over-constrain the system, and the coordinated MMSE approach where the leaked macrocell interference and femtocell interference is jointly suppressed performs better.

The remainder of the paper is organized as follows: In Section II, we introduce the system model. Interference alignment for macrocell users using successive SDP relaxations is presented in Section III. Section IV describes the precoding and decoding schemes for femtocell users. Numerical results are given in Section V. We conclude the paper in Section VI.

The notation used in the paper is as follows: We use lower (upper) bold case letters for vectors (matrices). $\mathbf{X}^{H}$ is used to denote the Hermitian transpose, $\mathbf{X}^{\dagger}$ as the pseudo-inverse of matrix $\mathbf{X}$, and $\otimes$ for the Kronecker product. $\|\cdot\|$ is the norm of a complex scalar or vector. Finally, $\operatorname{tr}(\mathbf{X})$ represents the trace of matrix $\mathbf{X}$, and $|\mathcal{S}|$ is the cardinality of the set $\mathcal{S}$.

\section{SySTEM MODEL}

The cellular network considered in this paper is the uplink of a co-existing macrocell-femtocell network with a single MBS at the center with $N_{o}$ receive antennas. Multiple FBSs are distributed over the macrocell coverage area ${ }^{1}$. The macrocell coverage area is partitioned into smaller areas of fixed radius in which the mobile users and FBSs are assumed to be able and willing to cooperate with each other. These clusters

\footnotetext{
${ }^{1}$ We treat inter-macrocell interference as noise and concentrate on one macrocell.
} 


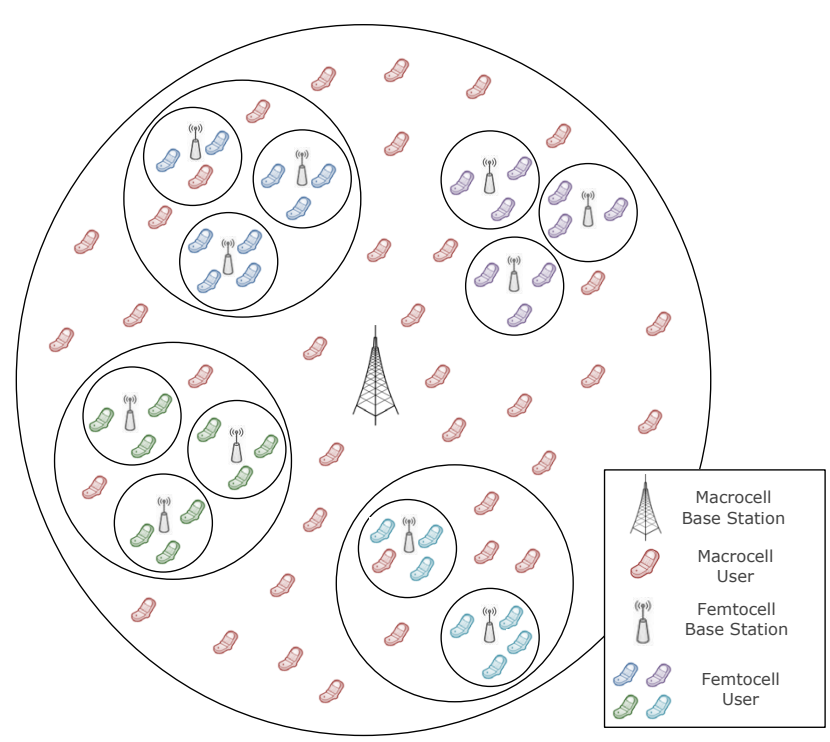

Fig. 1. System model with a single MBS and 4 femtocell clusters.

may represent a building or a neighborhood. We focus on a closed access model in the sense that the macrocell users' communication is facilitated by the MBS. An instance of the network model with 4 femtocell clusters is shown in Fig. 1.

We consider such a cluster with $F$ FBSs, $U_{f}$ FUs in the $f^{t h}$ femtocell and $M$ MUs. We assume $N_{t}$ transmit antennas at each mobile device, MU or FU, and $N_{f}$ receive antennas at the $f^{t h}$ FBS. The discrete time representation of the received signal at the $k^{t h} \mathrm{FBS}$ is then given as:

$\mathbf{y}_{k}=\underbrace{\sum_{i=1}^{U_{k}} \mathbf{H}_{k i}^{k} \mathbf{w}_{i}^{k} s_{i}^{k}}_{\substack{\text { signals from } k^{t h} \\ \text { femtocell users }}}+\underbrace{\sum_{m=1}^{M} \mathbf{H}_{k m}^{o} \mathbf{w}_{m}^{o} s_{m}^{o}}_{\text {macrocell interference }}+\underbrace{\sum_{\substack{f=1 \\ f \neq k}}^{F} \sum_{u=1}^{U_{f}} \mathbf{H}_{k u}^{f} \mathbf{w}_{u}^{f} s_{u}^{f}}_{\text {other femtocell interference }}+\mathbf{n}_{k}$

where $\mathbf{H}_{k m}^{o}$ represents the channel from the $m^{t h} \mathrm{MU}$ to the $k^{t h}$ FBS, and $\mathbf{H}_{k u}^{f}$ is the channel from the $u^{t h}$ user of the $f^{t h}$ femtocell to the $k^{t h}$ FBS. $\mathbf{w}_{u}^{f}$ denotes the precoding vector of the $u^{t h}$ user of the $f^{t h}$ femtocell, whereas $\mathbf{w}_{m}^{o}$ represents the precoding vector of the $m^{t h}$ MU. The number of femtocells in the macrocell coverage area is denoted by $F . s_{u}^{f}$ is the message of the $u^{t h}$ user of the $f^{t h}$ femtocell, and $s_{m}^{o}$ represents the message of the $m^{t h}$ MU. We assume the messages $s_{u}^{f}$ and $s_{m}^{o}= \pm 1$ with equal probability for $u=1, \ldots, U_{f}$, $f=1, \ldots, F$, and $m=1, \ldots, M$. The noise at the $k^{t h}$ FBS is denoted by $\mathbf{n}_{k}$, which consists of independent zero-mean Gaussian random variables with $E\left\{\mathbf{n}_{k} \mathbf{n}_{k}{ }^{H}\right\}=\sigma^{2} \mathbf{I}$. Similarly, the signal received at the MBS can be represented as:

$$
\mathbf{y}_{o}=\underbrace{\sum_{m=1}^{M} \mathbf{H}_{o m}^{o} \mathbf{w}_{m}^{o} s_{m}^{o}}_{\begin{array}{c}
\text { signals from } \\
\text { macrocell users }
\end{array}}+\underbrace{\sum_{f=1}^{F} \sum_{u=1}^{U_{f}} \mathbf{H}_{o u}^{f} \mathbf{w}_{u}^{f} s_{u}^{f}}_{\text {femtocell interference }}+\mathbf{n}_{o}
$$

where $\mathbf{H}_{o m}^{o}$ is used to denote the channel from the $m^{t h}$ MU to the MBS, and $\mathbf{H}_{o u}^{f}$ is the channel from the the $u^{t h}$ user of the $f^{t h}$ femtocell to the MBS. The noise at the MBS is $\mathbf{n}_{o}$, consisting of independent zero-mean Gaussian random variables with $E\left\{\mathbf{n}_{o} \mathbf{n}_{o}{ }^{H}\right\}=\sigma^{2} \mathbf{I}$. The channel state

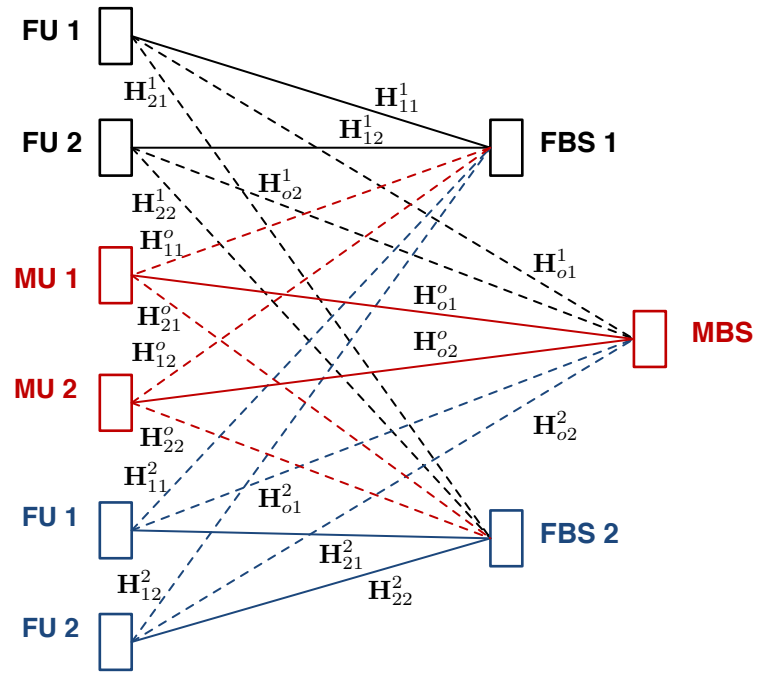

Fig. 2. Model for the case of 2 macrocell users and 2 FBSs, each with 2 users.

information (CSI) is known to the transmitter and receivers. An illustration of the system is provided in Fig. 2 for 2 MUs and 2 FBSs, each with 2 users.

Remark 1. Throughout the paper, we assume a single data stream per user for clarity of exposition. The proposed method can, however, be readily extended to multiple data streams per user and matrix precoders. For the sake of completeness, we provide the formulation of the SDP problem with SINR constraints with multiple data streams in the Appendix.

The inter-cluster interference in our model is managed separately for two scenarios. In a rural area, since the intercluster distances are much greater than the intra-femtocell distances within a cluster and users are sparsely distributed, intercluster interference is negligible due to low power femtocell users. In a dense urban environment, we employ interference avoidance for inter-cluster interference by assigning clusters to orthogonal frequency dimensions.

\section{Interference Alignment With SuCCESSIVE SDP RELAXATIONS}

In this section, we provide the conditions for aligning the cross-tier interference at multiple FBSs. We then formulate the minimum interference misalignment problem [21] at the femtocell tier as an SDP problem with minimum received SINR constraints for the MUs. Successive SDP relaxations are then utilized to determine the precoders of the MUs that minimize the interference misalignment at the FBSs while meeting the SINR requirements at the MBS. The dominant interference to femtocell uplinks is caused by a subset of the MUs closer to the FBSs. We propose to align all of these dominant interferer signals at the affected FBSs simultaneously. Consequently, the precoders of these users need to satisfy the following conditions: [21]:

$$
\begin{aligned}
& \mathbf{H}_{11}^{o} \mathbf{w}_{1}^{o}=\alpha_{12} \mathbf{H}_{12}^{o} \mathbf{w}_{2}^{o}=\cdots=\alpha_{1 M} \mathbf{H}_{1 M}^{o} \mathbf{w}_{M}^{o}, \\
& \mathbf{H}_{21}^{o} \mathbf{w}_{1}^{o}=\alpha_{22} \mathbf{H}_{22}^{o} \mathbf{w}_{2}^{o}=\cdots=\alpha_{2 M} \mathbf{H}_{2 M}^{o} \mathbf{w}_{M}^{o}, \\
& \quad \vdots \\
& \mathbf{H}_{F 1}^{o} \mathbf{w}_{1}^{o}=\alpha_{F 2} \mathbf{H}_{F 2}^{o} \mathbf{w}_{2}^{o}=\cdots=\alpha_{F M} \mathbf{H}_{F M}^{o} \mathbf{w}_{M}^{o},
\end{aligned}
$$


where $\alpha_{f m}$ is a constant, and the equations dictate that all interfering users span the same column space at each FBS for which they are a dominant interferer. That is, each interfering signal is represented by a linear combination of other interfering signals. Conditions (3)-(5) can be combined in a single matrix equation [21] by using the precoders and scaling coefficients:

$$
\mathbf{H w}=\mathbf{0}
$$

where $\mathbf{H}$ is an $\left(M \sum_{f=1}^{F} N_{f}\right) \times\left(M N_{t}\right)$ matrix with two nonzero matrix-elements in each row:

$$
\mathbf{H}=\left[\begin{array}{cccc}
\mathbf{H}_{11}^{o} & -\alpha_{12} \mathbf{H}_{12}^{o} & \ldots & \mathbf{0} \\
\vdots & \vdots & \ddots & \vdots \\
\mathbf{H}_{11}^{o} & \mathbf{0} & \ldots & -\alpha_{1 M} \mathbf{H}_{1 M}^{o} \\
\vdots & \vdots & \ddots & \vdots \\
\mathbf{H}_{F 1}^{o} & -\alpha_{F 2} \mathbf{H}_{F 2}^{o} & \ldots & \mathbf{0} \\
\vdots & \vdots & \ddots & \vdots \\
\mathbf{H}_{F 1}^{o} & \mathbf{0} & \ldots & -\alpha_{F M} \mathbf{H}_{F M}^{o}
\end{array}\right],
$$

and

$$
\mathbf{w}=\left[\begin{array}{llllll}
\mathbf{w}_{1}^{o T} & \mathbf{w}_{2}^{o T} & \mathbf{w}_{3}^{o T} & \ldots & \mathbf{w}_{M-1}^{o T} & \mathbf{w}_{M}^{o T}
\end{array}\right]^{T} .
$$

One approach for finding the interference aligning precoding matrices is to drive the norm of this expression as close to zero as possible as in (7), from which follows the notion of least squares approach for IA, proposed in [21]. We will follow this approach with a twist. Specifically, in addition to solving the least squares problem for IA, we also wish to ensure that the MUs do not fall below their SINR requirements as measured at the MBS. As a result, a new constrained optimization problem arises which we tackle by using semidefinite programming relaxations. That is to say, the norm minimization problem with individual minimum SINR constraints for each MU will incorporate successive SDP relaxations [22] and rank-one approximations.

We propose the following optimization problem to find the uplink MU precoders:

$$
\begin{array}{cl}
\underset{\mathbf{w}_{1}^{o}, \ldots, \mathbf{w}_{M}^{o}}{\min } & \|\mathbf{H w}\| \\
\text { s.t. } & S I N R_{i} \geq \gamma_{i} \\
& \left(\mathbf{w}_{i}^{o}\right)^{H} \mathbf{w}_{i}^{o} \leq \mathrm{P}_{i}^{o} \quad i=1, \ldots, M
\end{array}
$$

where $\mathrm{P}_{i}^{o}$ and $\gamma_{i}$ denote the maximum transmit power and minimum SINR threshold of MU $i$, respectively. We define the received SINR of MU $i$ at the MBS as:

$$
S I N R_{i}=\frac{\left(\mathbf{w}_{i}^{o}\right)^{H}\left(\mathbf{H}_{o i}^{o}\right)^{H} \mathbf{H}_{o i}^{o} \mathbf{w}_{i}^{o}}{\sum_{\substack{n=1 \\ n \neq i}}^{M}\left(\mathbf{w}_{n}^{o}\right)^{H}\left(\mathbf{H}_{o n}^{o}\right)^{H} \mathbf{H}_{o n}^{o} \mathbf{w}_{n}^{o}+\beta+\sigma^{2}}
$$

with

$$
\beta=\sum_{f=1}^{F} \sum_{u=1}^{U_{f}}\left(\mathbf{w}_{u}^{f}\right)^{H}\left(\mathbf{H}_{o u}^{f}\right)^{H} \mathbf{H}_{o u}^{f} \mathbf{w}_{u}^{f}
$$

where $\mathbf{H}_{\text {on }}^{o}$ denotes the channel from the $n^{\text {th }} \mathrm{MU}$ to the MBS, and $\sigma^{2}$ is the noise power. Observe that $\beta$ denotes the interference power from the FUs at the MBS.

Remark 2. Although $\beta$ depends on the choice of FU pre- coders, due to originating from a group of low power FUs, we shall assume that this dependence is not significant enough to warrant a joint optimization approach with the FU precoders ${ }^{2}$. We will verify this assumption numerically in Section $\mathrm{V}$ by comparing different initializations. We will thus use $\beta$ as an added noise term with variance equal to the average accumulated interference power from the FUs.

Using the conditions in (7), the problem can be re-written as:

$$
\begin{array}{cl}
\min _{\mathbf{w}_{1}^{o}, \ldots, \mathbf{w}_{M}^{o}} & \operatorname{tr}(\mathbf{R W}) \\
\text { s.t. } & \operatorname{tr}\left(\left(\widehat{\mathbf{R}}_{o i}-\gamma_{i} \sum_{n \neq i} \widehat{\mathbf{R}}_{o n}\right) \mathbf{W}\right) \geq \gamma_{i}\left(\sigma^{2}+\beta\right) \\
& \operatorname{tr}\left(\left(\operatorname{diag}\left(\mathbf{e}_{i}\right) \otimes \mathbf{I}_{\left(N_{t} \times N_{t}\right)}\right) \mathbf{W}\right) \leq \mathrm{P}_{i}^{o} \\
& \operatorname{rank}(\mathbf{W})=1 \\
& \mathbf{W} \succeq 0, \quad i=1, \ldots, M
\end{array}
$$

where $\mathbf{R}=\mathbf{H}^{H} \mathbf{H}, \mathbf{W}=\mathbf{w w}^{H}, \mathbf{R}_{\text {on }}=\left(\mathbf{H}_{\text {on }}^{o}\right)^{H} \mathbf{H}_{o n}^{o}, \widehat{\mathbf{R}}_{\text {on }}=$ $\operatorname{diag}\left(\mathbf{e}_{n}\right) \otimes \mathbf{R}_{\text {on }}$. The vector $\mathbf{e}_{n}=[0 \ldots 010 \ldots 0]^{T}$ is an $(M \times$ 1) unit vector with 1 as the $n^{\text {th }}$ element and zeros elsewhere. $\mathbf{I}_{\left(N_{t} \times N_{t}\right)}$ denotes the $\left(N_{t} \times N_{t}\right)$ identity matrix. By relaxing the rank-1 constraint, we obtain the semidefinite relaxation of the problem [23]:

$$
\begin{array}{ll}
\underset{\mathbf{w}_{1}^{o}, \ldots, \mathbf{w}_{M}^{o}}{\operatorname{minimize}} & \operatorname{tr}(\mathbf{R W}) \\
\text { subject to } & \operatorname{tr}\left(\left(\widehat{\mathbf{R}}_{o i}-\gamma_{i} \sum_{n \neq i} \widehat{\mathbf{R}}_{o n}\right) \mathbf{W}\right) \geq \gamma_{i}\left(\sigma^{2}+\beta\right) \\
& \operatorname{tr}\left(\left(\operatorname{diag}\left(\mathbf{e}_{i}\right) \otimes \mathbf{I}_{\left(N_{t} \times N_{t}\right)}\right) \mathbf{W}\right) \leq \mathrm{P}_{i}^{o} \\
& \mathbf{W} \succeq 0, \quad i=1, \ldots, M
\end{array}
$$

The SDP in (11) can be solved effectively, for instance by using SeDuMi [24]. In case the resulting solution has a higher rank than one, we can use the eigenvector approximation in [25], in which the vector $\mathbf{w}$ is approximated as the eigenvector $\mathbf{q}_{1}$ corresponding to the largest eigenvalue of $\mathbf{W}$, scaled by the square root of the largest eigenvalue of $\mathbf{W}, \lambda_{1}$, i.e.,

$$
\begin{aligned}
\mathbf{W} & =\mathbf{w w}^{H}=\sum_{i} \lambda_{i} \mathbf{q}_{i} \mathbf{q}_{i}^{H} \\
\mathbf{w} & \cong \sqrt{\lambda_{1}} \mathbf{q}_{1} .
\end{aligned}
$$

Following this step, the coefficients are determined from conditions (3)-(5) [21], as given by:

$$
\begin{aligned}
\alpha_{k m} & =\left(\mathbf{H}_{k m}^{o} \mathbf{w}_{m}^{o}\right)^{\dagger}\left(\mathbf{H}_{k 1}^{o} \mathbf{w}_{1}^{o}\right) \\
\left(\mathbf{H}_{k m}^{o} \mathbf{w}_{m}^{o}\right)^{\dagger} & =\left(\left(\mathbf{H}_{k m}^{o} \mathbf{w}_{m}^{o}\right)^{H}\left(\mathbf{H}_{k m}^{o} \mathbf{w}_{m}^{o}\right)\right)^{-1}\left(\mathbf{H}_{k m}^{o} \mathbf{w}_{m}^{o}\right)^{H}
\end{aligned}
$$

Remark 3. (Feasibility of SDP) The objective function of the SDP problem in (11) is always bounded below by 0 . As a result, a solution exists for the successive SDP relaxations as long as the feasible set is not empty, that is, the SINR constraints are achievable for the given channel configurations and the maximum transmit power constraints; see also Section IV.C.

\footnotetext{
${ }^{2}$ The design of these precoders does have a significant impact on the performance of the FU themselves and will be addressed in Section IV.
} 
Remark 4. (Feasibility of Perfect Interference Alignment) Feasibility of perfect IA has recently been considered in the context of $K$-user interference channels [26], [27], for which the IA conditions form a multivariate polynomial system. In our formulation, the IA condition is equivalent to solving the linear matrix equation in (6). The number of rows of $\mathbf{H}$ in (6) is:

$$
\mathcal{N}_{\text {row }}=M \sum_{f=1}^{F} N_{f}
$$

which corresponds to the number of equations to be solved. The number of columns $\mathcal{N}_{\text {col }}$ is given as:

$$
\mathcal{N}_{\text {col }}=M N_{t}
$$

which is equal to the number of variables. Then the linear system is overdetermined when

$$
\mathcal{N}_{\text {row }}>\mathcal{N}_{\text {col }} \Rightarrow M \sum_{f=1}^{F} N_{f}>M N_{t} .
$$

It is known that an exact solution does not exist for such systems, and that perfect IA is not feasible. That is why we apply the least squares approach to minimize the unaligned interference.

\section{Precoder And Decoder Design for Femtocell USERS}

In the previous sections, we have designed the MU precoders so that their interfering signals are aligned at the FBS, while keeping their SINR levels. In this section, we will design the FU precoders and decoders.

\section{A. Coordinated MMSE Approach}

Femtocell users can either cooperate and contribute to interference alignment, which increases the system complexity and the load on the backhaul or they can try to improve their own performance by interference cancellation. We opt for the latter (see also Remark 2), and apply MMSE precoding/decoding for the FUs, while considering the aligned interference received from the MUs. The decision statistic for the estimated bit of the $j^{\text {th }}$ user of the $k^{\text {th }}$ femtocell is:

$$
\begin{aligned}
\hat{s}_{j}^{k}= & \sum_{i=1}^{U_{k}}\left(\mathbf{g}_{j}^{k}\right)^{H} \mathbf{H}_{k i}^{k} \mathbf{w}_{i}^{k} s_{i}^{k}+\sum_{m=1}^{M}\left(\mathbf{g}_{j}^{k}\right)^{H} \mathbf{H}_{k m}^{o} \mathbf{w}_{m}^{o} s_{m}^{o} \\
& +\sum_{\substack{f=1 \\
f \neq k}}^{F} \sum_{u=1}^{U_{f}}\left(\mathbf{g}_{j}^{k}\right)^{H} \mathbf{H}_{k u}^{f} \mathbf{w}_{u}^{f} s_{u}^{f}+\left(\mathbf{g}_{j}^{k}\right)^{H} \mathbf{n}_{k}
\end{aligned}
$$

where $\mathbf{g}_{j}^{k}$ is the decoding vector for the $j^{\text {th }}$ user of the $k^{t h}$ femtocell. Using the conditions in (3)-(5) and (19), the minimum sum MSE at the $k^{\text {th }}$ FBS can be formulated as:

$$
\underset{\substack{\mathbf{w}_{1}^{k}, \ldots, \mathbf{w}_{U_{k}}^{k} \\ \mathbf{g}_{1}^{k}, \ldots, \mathbf{g}_{U_{k}}^{k}}}{\operatorname{minimize}} \sum_{j=1}^{U_{k}} E\left\{\left\|\hat{s}_{j}^{k}-s_{j}^{k}\right\|^{2}\right\}
$$

subject to $\left(\mathbf{w}_{j}^{k}\right)^{H} \mathbf{w}_{j}^{k} \leq \mathrm{P}_{j}^{k} \quad j=1, \ldots, U_{k}$

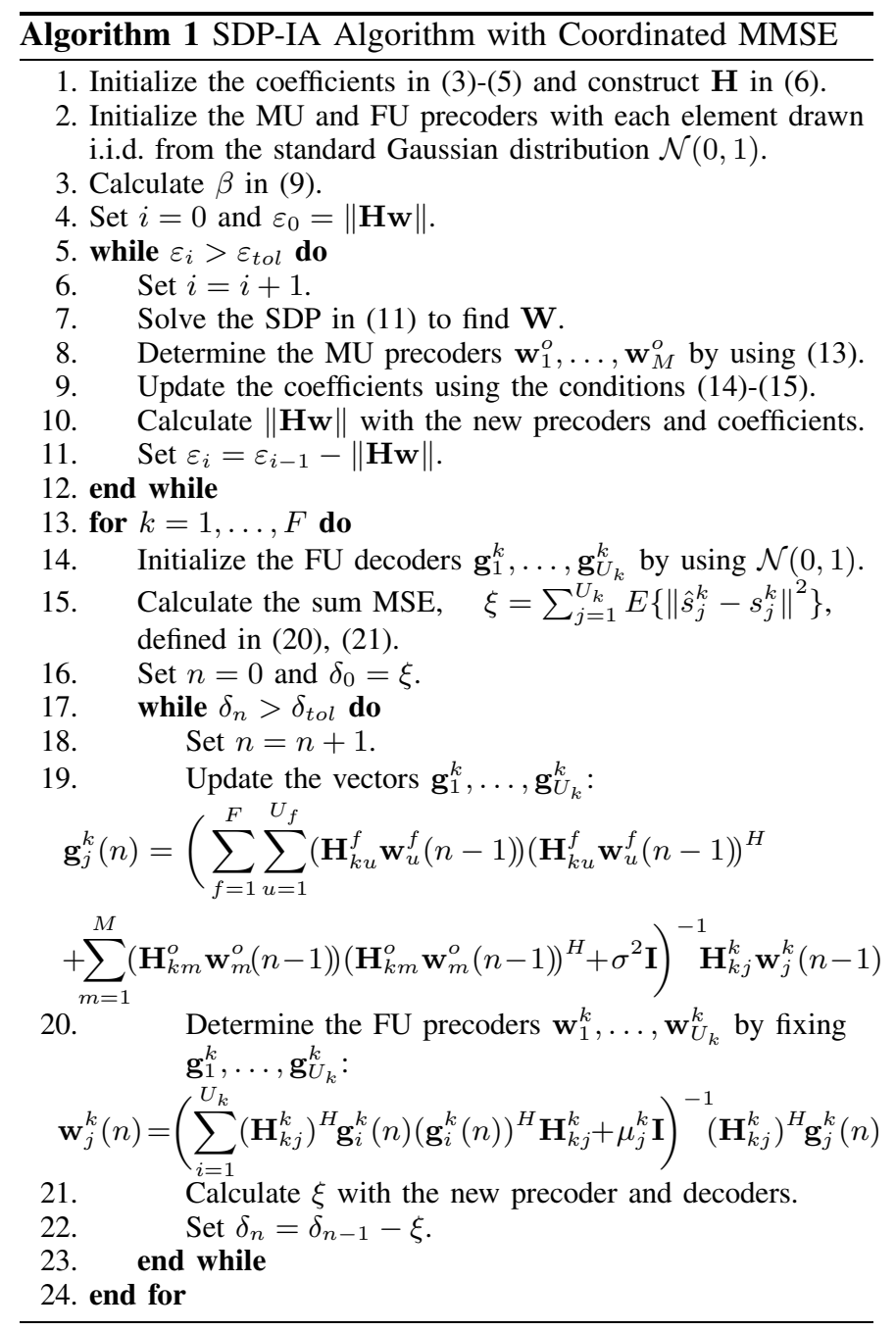

We can also express (20) as:

$$
\begin{array}{ll}
\underset{\substack{\mathbf{w}_{1}^{k}, \ldots, \mathbf{w}_{U_{k}}^{k} \\
\mathbf{g}_{1}^{k}, \ldots, \mathbf{g}_{U_{k}}^{k}}}{\operatorname{minimize}} & \sum_{j=1}^{U_{k}}\left[\left\|\left(\mathbf{g}_{j}^{k}\right)^{H} \mathbf{H}_{k j}^{k} \mathbf{w}_{j}^{k}-1\right\|^{2}\right. \\
& +\sum_{\substack{i=1 \\
i \neq j}}^{U_{k}}\left\|\left(\mathbf{g}_{j}^{k}\right)^{H} \mathbf{H}_{k i}^{k} \mathbf{w}_{i}^{k}\right\|^{2}+\sum_{m=1}^{M}\left\|\left(\mathbf{g}_{j}^{k}\right)^{H} \mathbf{H}_{k m}^{o} \mathbf{w}_{m}^{o}\right\|^{2} \\
& \left.+\sum_{\substack{f=1 \\
f \neq k}}^{F} \sum_{u=1}^{U_{f}}\left\|\left(\mathbf{g}_{j}^{k}\right)^{H} \mathbf{H}_{k u}^{f} \mathbf{w}_{u}^{f}\right\|^{2}+\left\|\mathbf{g}_{j}^{k}\right\|^{2} \sigma^{2}\right] \\
\text { subject to } \quad\left(\mathbf{w}_{j}^{k}\right)^{H} \mathbf{w}_{j}^{k} \leq \mathrm{P}_{j}^{k} \quad j=1, \ldots, U_{k}
\end{array}
$$

where $\mathrm{P}_{j}^{k}$ is the maximum transmit power of the $j^{\text {th }}$ user of the $k^{t h}$ femtocell.

The problem in (21) is jointly convex in $\mathbf{w}_{j}^{k}, j=1, \ldots, U_{k}$, if all $\mathbf{g}_{j}^{k}$ are fixed, and jointly convex in $\mathbf{g}_{j}^{k}, j=1, \ldots, U_{k}$, if all $\mathbf{w}_{j}^{k}$ are fixed. Thus, we can design an iterative algorithm by first fixing the decoding vectors and obtaining the precoding vectors, then fixing the precoding vectors to obtain the decoding vectors. Such an iterative procedure for obtaining the precoders and decoders is used in [28] for a multiple access MIMO channel. The resulting precoders-decoders are 
guaranteed to converge to a local optimum which applies in the present setting as well. When the vectors $\mathbf{g}_{j}^{k}$ are fixed, the resulting optimization problem is over the precoders $\mathbf{w}_{j}^{k}$, $j=1, \ldots, U_{k}$, can be written as follows:

$$
\begin{aligned}
\underset{\mathbf{w}_{1}^{k}, \ldots, \mathbf{w}_{U_{k}}^{k}}{\operatorname{minimize}} & \sum_{j=1}^{U_{k}}\left[\left\|\left(\mathbf{g}_{j}^{k}\right)^{H} \mathbf{H}_{k j}^{k} \mathbf{w}_{j}^{k}-1\right\|^{2}+\sum_{\substack{i=1 \\
i \neq j}}^{U_{k}}\left\|\left(\mathbf{g}_{j}^{k}\right)^{H} \mathbf{H}_{k i}^{k} \mathbf{w}_{i}^{k}\right\|^{2}\right. \\
& +\sum_{m=1}^{M}\left\|\left(\mathbf{g}_{j}^{k}\right)^{H} \mathbf{H}_{k m}^{o} \mathbf{w}_{m}^{o}\right\|^{2}+\sum_{\substack{f=1 \\
f \neq k}}^{F} \sum_{u=1}^{U_{f}}\left\|\left(\mathbf{g}_{j}^{k}\right)^{H} \mathbf{H}_{k u}^{f} \mathbf{w}_{u}^{f}\right\|^{2} \\
& \left.+\left\|\mathbf{g}_{j}^{k}\right\|^{2} \sigma^{2}\right]
\end{aligned}
$$

subject to $\left(\mathbf{w}_{j}^{k}\right)^{H} \mathbf{w}_{j}^{k} \leq \mathrm{P}_{j}^{k} \quad j=1, \ldots, U_{k}$

The KKT conditions for (22) are:

\section{Stationarity:}

$$
\sum_{i=1}^{U_{k}}\left(\mathbf{H}_{k j}^{k}\right)^{H} \mathbf{g}_{i}^{k}\left(\mathbf{g}_{i}^{k}\right)^{H} \mathbf{H}_{k j}^{k} \mathbf{w}_{j}^{k^{*}}+\mu_{j}^{k} \mathbf{w}_{j}^{k^{*}}-\left(\mathbf{H}_{k j}^{k}\right)^{H} \mathbf{g}_{j}^{k}=0
$$

Complementary Slackness: $\quad \mu_{j}^{k}\left(\left(\mathbf{w}_{j}^{k^{*}}\right)^{H} \mathbf{w}_{j}^{k^{*}}-P_{j}^{k}\right)=0$

Dual feasibility:

$$
\mu_{j}^{k} \geq 0
$$

Primary feasibility:

$$
\left(\mathbf{w}_{j}^{k^{*}}\right)^{H} \mathbf{w}_{j}^{k^{*}} \leq P_{j}^{k}
$$

where $j=1, \ldots, U_{k}$, and $\mathbf{w}_{j}^{k^{*}}$ denotes the optimal value for $\mathbf{w}_{j}^{k}$. Using the KKT conditions in (23), we can obtain the optimal precoding vectors for (22) as:

$$
\mathbf{w}_{j}^{k^{*}}=\left(\sum_{i=1}^{U_{k}}\left(\mathbf{H}_{k j}^{k}\right)^{H} \mathbf{g}_{i}^{k}\left(\mathbf{g}_{i}^{k}\right)^{H} \mathbf{H}_{k j}^{k}+\mu_{j}^{k} \mathbf{I}\right)^{-1}\left(\mathbf{H}_{k j}^{k}\right)^{H} \mathbf{g}_{j}^{k}
$$

where $\mu_{j}^{k}$ is determined to satisfy the transmit power constraint, i.e., $\left(\mathbf{w}_{j}^{k}\right)^{H} \mathbf{w}_{j}^{k}=\mathrm{P}_{j}^{k}$. Similarly, we fix the vectors $\mathbf{w}_{j}^{k}$ for $j=1, \ldots, U_{k}$ and obtain the KKT conditions for the resulting problem, from which the optimal decoding vector for fixed precoders follows:

$$
\begin{aligned}
\mathbf{g}_{j}^{k^{*}}=\left(\sum_{f=1}^{F}\right. & \sum_{u=1}^{U_{f}}\left(\mathbf{H}_{k u}^{f} \mathbf{w}_{u}^{f}\right)\left(\mathbf{H}_{k u}^{f} \mathbf{w}_{u}^{f}\right)^{H} \\
& \left.+\sum_{m=1}^{M}\left(\mathbf{H}_{k m}^{o} \mathbf{w}_{m}^{o}\right)\left(\mathbf{H}_{k m}^{o} \mathbf{w}_{m}^{o}\right)^{H}+\sigma^{2} \mathbf{I}\right)^{-1} \mathbf{H}_{k j}^{k} \mathbf{w}_{j}^{k}
\end{aligned}
$$

for $j=1, \ldots, U_{k}$. Equations (24) and (25) together yield one iteration of alternating optimization. The SDP-IA algorithm with coordinated MMSE, putting together our findings in this section with that of Section III, is presented in Algorithm 1. In the implementation of the algorithm, SDP-IA iterations terminate when the improvements in the objective function Hw is less than the tolerance level $\varepsilon_{t o l}$. Similarly, MMSE stage stops whenever the improvement in the sum MSE is less than $\delta_{t o l}$.

\section{B. Coordinated Zero-Forcing Approach}

In this section, we consider the scheme in which each FBS zero-forces the aligned macrocell interference in addition to minimizing the sum MSE of its own users ${ }^{3}$. A coordinated zero-forcing beamforming was used for SINR maximization in [30], with ideas from [31].

Using (3)-(5) and (19), we can formulate the problem at FBS $k$ as:

$$
\begin{array}{cl}
\min _{\substack{\mathbf{w}_{1}^{k}, \ldots, \mathbf{w}_{U_{k}}^{k} \\
\mathbf{g}_{1}^{k}, \ldots, \mathbf{g}_{U_{k}}^{k}}} & \sum_{j=1}^{U_{k}}\left[\left\|\left(\mathbf{g}_{j}^{k}\right)^{H} \mathbf{H}_{k j}^{k} \mathbf{w}_{j}^{k}-1\right\|^{2}+\sum_{\substack{i=1 \\
i \neq j}}^{U_{k}}\left\|\left(\mathbf{g}_{j}^{k}\right)^{H} \mathbf{H}_{k i}^{k} \mathbf{w}_{i}^{k}\right\|^{2}\right. \\
& \left.+\sum_{\substack{f=1 \\
f \neq k}}^{F} \sum_{u=1}^{U_{f}}\left\|\left(\mathbf{g}_{j}^{k}\right)^{H} \mathbf{H}_{k u}^{f} \mathbf{w}_{u}^{f}\right\|^{2}+\left\|\mathbf{g}_{j}^{k}\right\|^{2} \sigma^{2}\right] \\
\text { subject to } & \left(\mathbf{g}_{j}^{k}\right)^{H} \mathbf{H}_{k 1}^{o} \mathbf{w}_{1}^{o}=0 \\
& \left(\mathbf{w}_{j}^{k}\right)^{H} \mathbf{w}_{j}^{k} \leq \mathrm{P}_{j}^{k} \quad j=1, \ldots, U_{k}
\end{array}
$$

where $\mathrm{P}_{j}^{k}$ denotes the maximum transmit power of the $j^{t h}$ user of femtocell $k$. The zero-forcing constraint in (26) implies that $\mathbf{g}_{j}^{k}$ should be in the null space of $\left(\mathbf{H}_{k 1}^{o} \mathbf{w}_{1}^{o}\right)$ [32], from which we can define a decoding vector as:

$$
\mathbf{g}_{j}^{k}=\mathbf{U}_{k}^{0} \mathbf{v}_{j}^{k}
$$

where $\left[\mathbf{U}_{k}^{0} \mathbf{U}_{k}^{1}\right] \boldsymbol{\Lambda}_{k} \mathbf{V}_{k}$ is obtained from the SVD of $\mathbf{H}_{k 1}^{o} \mathbf{w}_{1}^{o}$ and the columns of $\mathbf{U}_{k}^{0}$ is a nullspace basis of $\mathbf{H}_{k 1}^{o} \mathbf{w}_{1}^{o}$. Letting $\left(\mathbf{U}_{k}^{0}\right)^{H} \mathbf{H}_{k j}^{k}=\widetilde{\mathbf{H}}_{k j}^{k}$, the problem in (26) is equivalent to:

$$
\begin{aligned}
\min _{\substack{\mathbf{w}_{1}^{k}, \ldots, \mathbf{w}_{U_{k}}^{k} \\
\mathbf{v}_{1}^{k}, \ldots, \mathbf{v}_{U_{k}}^{k}}} \sum_{j=1}^{U_{k}}\left[\left\|\left(\mathbf{v}_{j}^{k}\right)^{H} \widetilde{\mathbf{H}}_{k j}^{k} \mathbf{w}_{j}^{k}-1\right\|^{2}+\sum_{\substack{i=1 \\
i \neq j}}^{U_{k}}\left\|\left(\mathbf{v}_{j}^{k}\right)^{H} \widetilde{\mathbf{H}}_{k i}^{k} \mathbf{w}_{i}^{k}\right\|^{2}\right. \\
\left.+\sum_{\substack{f=1 \\
f \neq k}}^{F} \sum_{u=1}^{U_{f}}\left\|\left(\mathbf{v}_{j}^{k}\right)^{H} \widetilde{\mathbf{H}}_{k u}^{f} \mathbf{w}_{u}^{f}\right\|^{2}+\left\|\mathbf{v}_{j}^{k}\right\|^{2} \sigma^{2}\right]
\end{aligned}
$$$$
\text { subject to }\left(\mathbf{w}_{j}^{k}\right)^{H} \mathbf{w}_{j}^{k} \leq \mathrm{P}_{j}^{k} \quad j=1, \ldots, U_{k}
$$

Equation (28) is convex in $\mathbf{w}_{j}^{k}$ when all $\mathbf{v}_{j}^{k}$ are fixed, and convex in $\mathbf{v}_{j}^{k}$ when all $\mathbf{w}_{j}^{k}$ are fixed. Thus, once again, we can utilize alternating minimization to obtain an iterative algorithm by first fixing the decoding matrices and determining the precoding matrices, then fixing the precoding matrices to obtain the decoding matrices. When decoding matrices in (28) are fixed, the vectors $\mathbf{v}_{j}^{k}$ are fixed as a consequence, and the resulting problem can be written as follows:

$$
\begin{array}{cc}
\min _{\mathbf{w}_{1}^{k}, \ldots, \mathbf{w}_{U_{k}}^{k}} & \sum_{j=1}^{U_{k}}\left[\left\|\left(\mathbf{v}_{j}^{k}\right)^{H} \widetilde{\mathbf{H}}_{k j}^{k} \mathbf{w}_{j}^{k}-1\right\|^{2}+\sum_{\substack{i=1 \\
i \neq j}}^{U_{k}}\left\|\left(\mathbf{v}_{j}^{k}\right)^{H} \widetilde{\mathbf{H}}_{k i}^{k} \mathbf{w}_{i}^{k}\right\|^{2}\right. \\
& \left.+\sum_{\substack{f=1 \\
f \neq k}}^{F} \sum_{u=1}^{U_{f}}\left\|\left(\mathbf{v}_{j}^{k}\right)^{H} \widetilde{\mathbf{H}}_{k u}^{f} \mathbf{w}_{u}^{f}\right\|^{2}+\left\|\mathbf{v}_{j}^{k}\right\|^{2} \sigma^{2}\right] \\
\text { subject to } \quad\left(\mathbf{w}_{j}^{k}\right)^{H} \mathbf{w}_{j}^{k} \leq \mathrm{P}_{j}^{k} \quad j=1, \ldots, U_{k}
\end{array}
$$

${ }^{3}$ This approach represents the part presented at Globecom 2011 [29]. 
The KKT conditions for (29) can be determined as:

Stationarity:

$$
\begin{array}{ll}
\sum_{i=1}^{U_{k}}\left(\widetilde{\mathbf{H}}_{k j}^{k}\right)^{H} \mathbf{v}_{i}^{k}\left(\mathbf{v}_{i}^{k}\right)^{H} \widetilde{\mathbf{H}}_{k j}^{k} \mathbf{w}_{j}^{k^{*}}+\mu_{j}^{k} \mathbf{w}_{j}^{k^{*}}-\left(\widetilde{\mathbf{H}}_{k j}^{k}\right)^{H} \mathbf{v}_{j}^{k}=0 \\
\text { Complementary Slackness: } & \mu_{j}^{k}\left(\left(\mathbf{w}_{j}^{k^{*}}\right)^{H} \mathbf{w}_{j}^{k^{*}}-P_{j}^{k}\right)=0 \\
\text { Dual feasibility: } & \mu_{j}^{k} \geq 0 \\
\text { Primary feasibility: } & \left(\mathbf{w}_{j}^{k^{*}}\right)^{H} \mathbf{w}_{j}^{k^{*}} \leq P_{j}^{k}
\end{array}
$$

where $j=1, \ldots, U_{k}$, and $\mathbf{w}_{j}^{k^{*}}$ denotes the optimal value for $\mathbf{w}_{j}^{k}$. Using the KKT conditions in (30), we can obtain the optimal precoding vectors for fixed decoders as:

$$
\mathbf{w}_{j}^{k^{*}}=\left(\sum_{i=1}^{U_{k}}\left(\widetilde{\mathbf{H}}_{k j}^{k}\right)^{H} \mathbf{v}_{i}^{k}\left(\mathbf{v}_{i}^{k}\right)^{H} \widetilde{\mathbf{H}}_{k j}^{k}+\mu_{j}^{k} \mathbf{I}\right)^{-1}\left(\widetilde{\mathbf{H}}_{k j}^{k}\right)^{H} \mathbf{v}_{j}^{k}
$$

with $\mu_{j}^{k}$ determined such that $\left(\mathbf{w}_{j}^{k}\right)^{H} \mathbf{w}_{j}^{k}=\mathrm{P}_{j}^{k}$. Similarly, we fix the precoders in (28) to obtain:

$$
\mathbf{v}_{j}^{k^{*}}=\left(\sum_{f=1}^{F} \sum_{u=1}^{U_{f}}\left(\widetilde{\mathbf{H}}_{k u}^{f} \mathbf{w}_{u}^{f}\right)\left(\widetilde{\mathbf{H}}_{k u}^{f} \mathbf{w}_{u}^{f}\right)^{H}+\sigma^{2} \mathbf{I}\right)^{-1} \widetilde{\mathbf{H}}_{k j}^{k} \mathbf{w}_{j}^{k}
$$

Thus, (31) and (32) together constitute one iteration of the minimum sum MSE with coordinated zero-forcing method. This method is employed with MU precoder design from Section III to construct the SDP-IA with coordinated zeroforcing algorithm presented in Algorithm 2.

\section{Convergence of the Two-Tier Interference Management Scheme}

In this section, we discuss the convergence of the proposed two-tier iterative interference management schemes. We start by considering the first stage, which aligns the macrocell interference at the FBSs by designing the MU precoders. Convergence of successive semidefinite relaxations has been shown in [22] from which we know that the objective function which represents the amount of interference misalignment reduces at each iteration. Since the term $\|\mathbf{H w}\|$ is also bounded below by zero, we can conclude that whenever the constraints are feasible due to the channel configurations and the minimum SINR constraints, the IA part of the algorithm converges. We note that although the SDP relaxation is an approximation to the original IA problem, the simulation results suggest that the remnant interference after convergence is negligible. After the precoders of the MUs are determined, the precoders-decoders of the FUs are obtained iteratively by solving a problem of minimizing the sum MSE at each FBS. We know that the objective function, sum MSE, is again bounded below by zero and is decreasing at each iteration, thus we conclude that the second stage of the problem, i.e., coordinated MMSE part, also converges. The coordinated zero-forcing algorithm, which also minimizes the sum MSE, converges following a similar argument. Thus we conclude that the SDP-IA algorithm, either with coordinated MMSE or zero-forcing, is convergent whenever the individual problems are feasible with the given channels, SINR requirements and maximum power constraints.

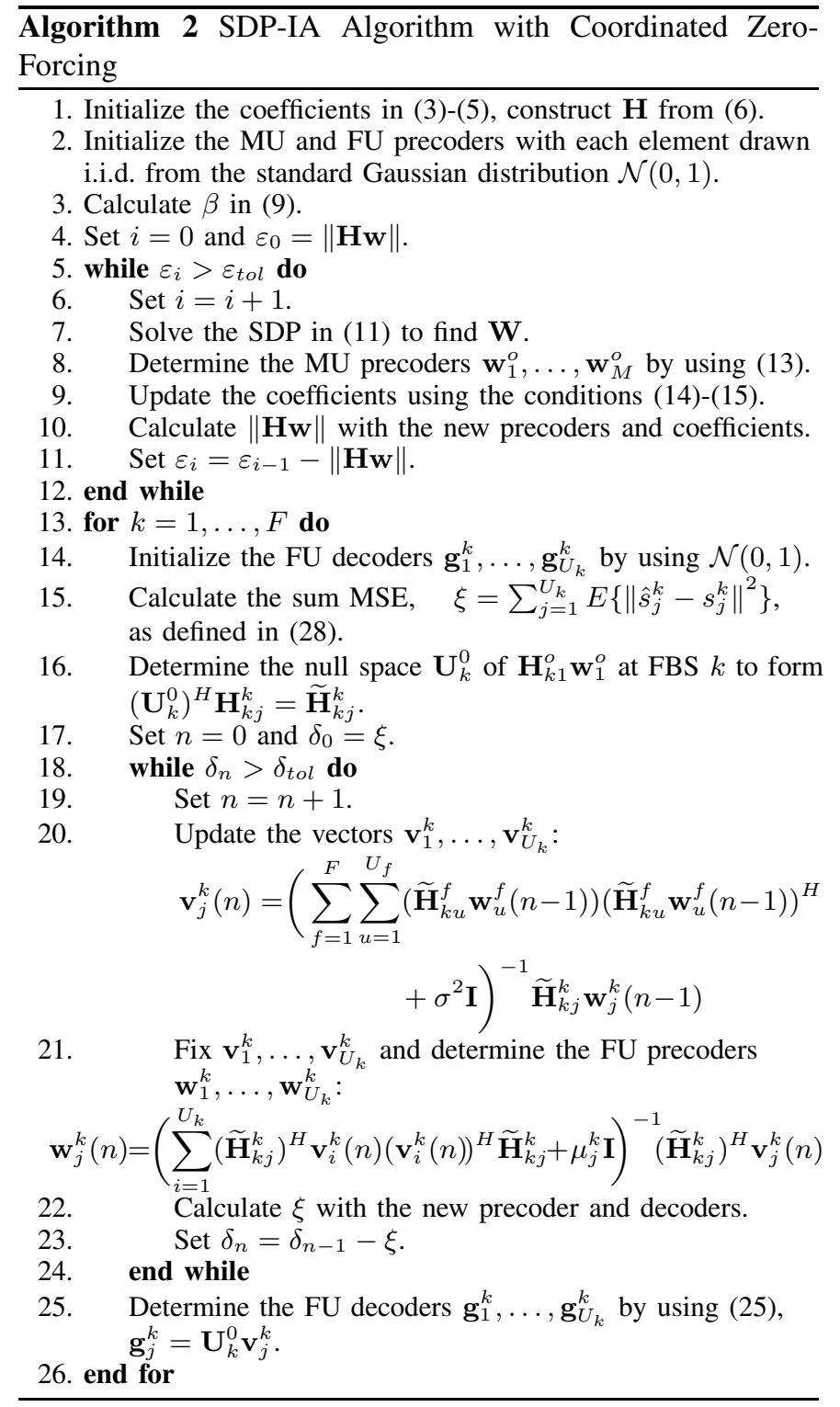

\section{NUMERICAL RESUltS}

Simulations are performed to compare the performance of the proposed SDP-IA macrocell IA algorithms (Algorithm 1 and Algorithm 2) with a baseline setting in which MUs minimize their sum MSE at the MBS, without regard to the FUs. Simulations use Rayleigh fading channels with the path loss modeled according to the ITU-R channel model [33] specifications for femtocell and macrocell users. Noise power is at $-110 \mathrm{~dB}$. Power control at both MBS and FBSs is employed to compensate for the path loss. The maximum transmit power is $1 \mathrm{~W}$ and $1 \mathrm{~mW}$ for the MUs and FUs, respectively.

We discuss two scenarios. First one is a suburban two-tier cellular network with a MBS that has a coverage radius of $2 \mathrm{~km}$. A circular area with a radius of $150 \mathrm{~m}$, denoting the group of FBSs close to each other, is placed according to a uniform distribution within the macrocell coverage area. MUs residing in this area are to be aligned at every FBS within the group. 3 FBSs, each having 3 users and a coverage radius of $30 \mathrm{~m}$, are placed using a uniform random distribution over the 


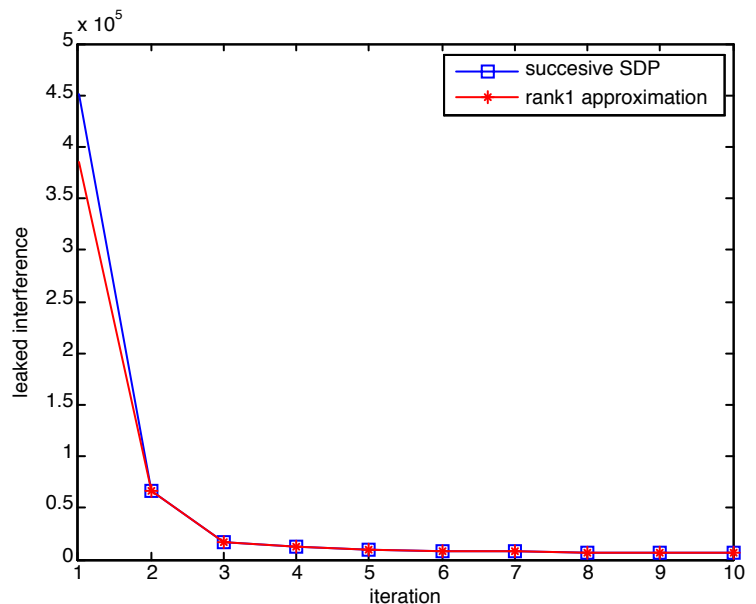

Fig. 3. Convergence results of the SDP-IA algorithm.

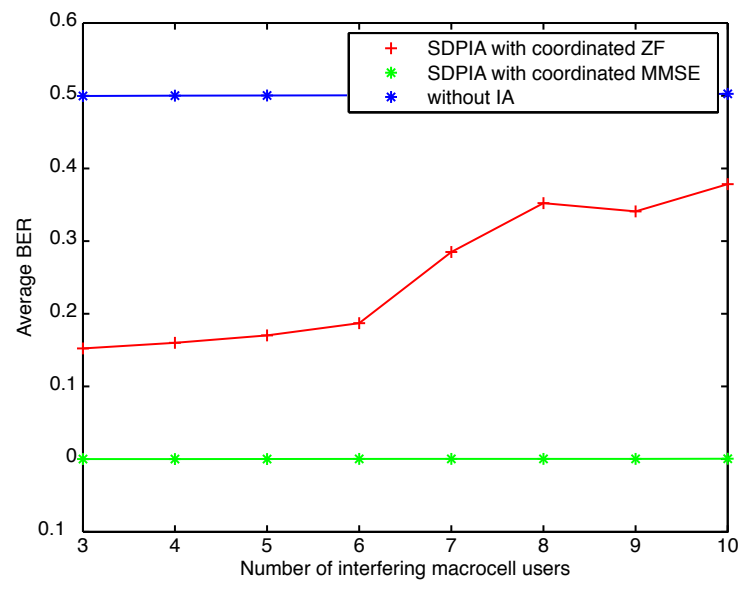

Fig. 4. Average BER of the femtocell users with and without SDP-IA algorithm.

circular area. FBSs have 4 receive antennas, and each mobile user, MU or FU, has 4 transmit antennas.

The convergence of the SDP-IA algorithm for 10 MUs and a minimum SINR requirement of 0.1 at the MBS is presented in Fig. 3, both with and without the rank-1 approximation. The comparison of SDP-IA with coordinated MMSE, SDPIA with coordinated zero forcing, and the case when no IA is applied and MUs aim to maximize their own performance at the MBS, is given in Fig. 4 in terms of average BER versus the number of MUs interfering to the femtocell cluster. The figure demonstrates that IA improves the average BER compared to the no-IA scheme, and that the performance of the coordinated MMSE approach is better than the coordinated zero-forcing scheme. In effect, the additional zero-forcing ends up overconstraining the transceivers of FUs. By contrast, coordinated MMSE manages the leaked interference from MUs jointly with the femtocell interference. The number of MUs that can be aligned via the SDP-IA algorithm for different minimum SINR requirements at the MBS is depicted in Fig. 5.

The average BER of the FUs with respect to the number of interfering MUs for the SDP-IA with coordinated MMSE

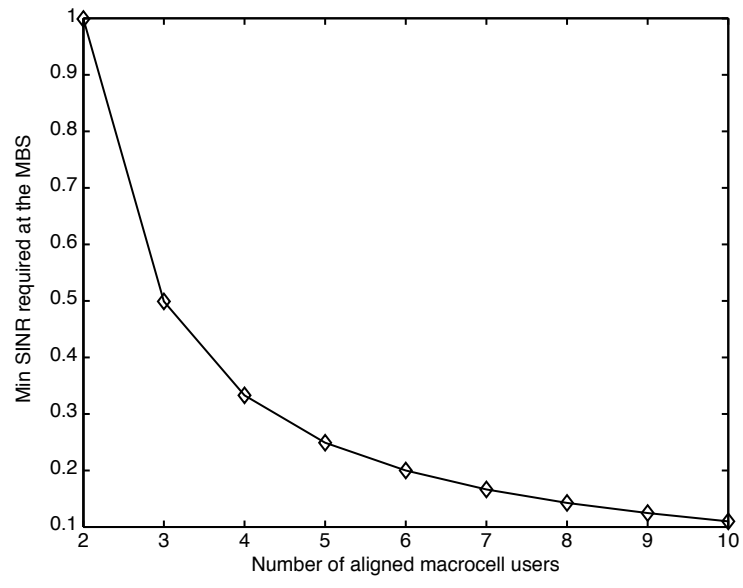

Fig. 5. Number of macrocell users that can be aligned subject to min SINR requirement at the MBS.

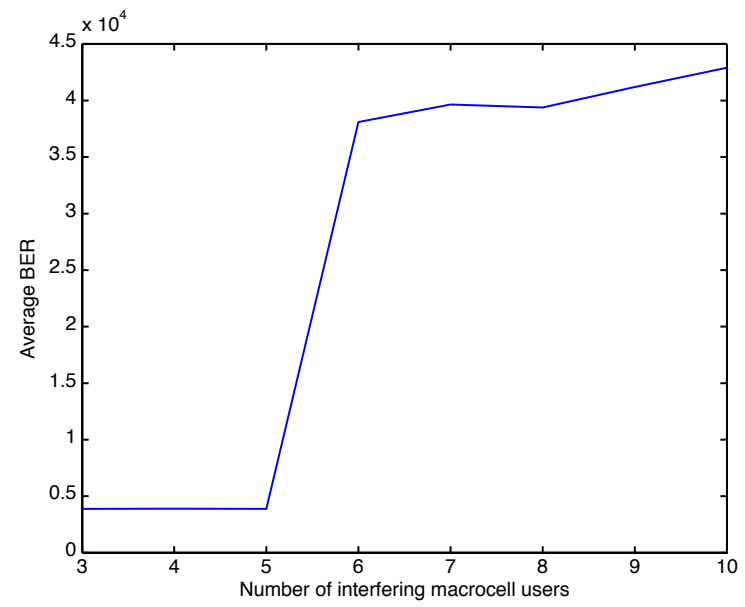

Fig. 6. Average BER of the femtocell users with SDP-IA algorithm with MMSE precoding/decoding for femtocell users.

algorithm is given in Fig. 6, for a single femtocell cluster. From Fig. 6 it can be seen that the average BER of the FUs have decreased, correspondingly their performances have improved.

We present the impact of aggregate femtocell interference on the received SINR of each MU for various SINR constraints in Fig. 7, by varying the number of MUs in each cluster to achieve different ratios of MU and FUs as the rationale for Remark 2. In this figure, FU precoders are either chosen randomly (which will not necessarily perform well for FUs), or optimized iteratively in a joint fashion with the MBS precoders. Fig. 7 suggests that the aggregate interference power from FUs on the MUs is small, no matter how the FU tranceivers are chosen. This affirms our view on the asymmetry of the two-tier interference and allows us to utilize the two stage interference management scheme for the design of FU and MU precoders instead of tackling jointly which would have necessitated a fully centralized implementation of the two tiers.

The second scenario we study is a dense urban cellular 


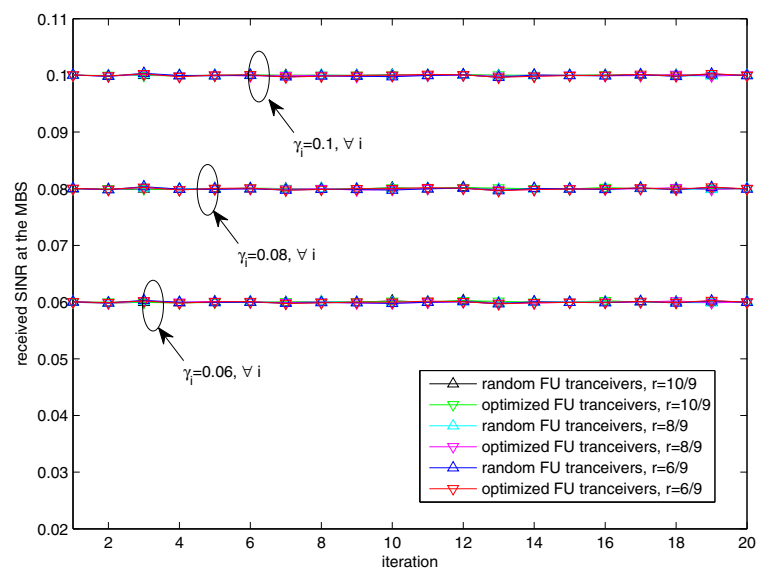

Fig. 7. The effect of aggregate femtocell user power to the received SINR at the MBS vs. different channel realizations, $r$ denotes the ratio of the macrocell interferers to femtocell users.

system. In this model, the macrocell coverage area, a circle with a $300 \mathrm{~m}$ radius, is partitioned into 7 hexagonal cells using disjoint sets of orthogonal dimensions. Macrocell and femtocell users that reside within a cell, i.e., orthogonal dimension group, share a set of orthogonal dimensions separate from other cells. These orthogonal dimensions can be in terms of frequency and time dimensions and do not interfere with each other. The hexagonal cells are approximated by a circular area with a radius of $100 \mathrm{~m}$. The system involves the outdoor MUs which are denoted as pedestrians as well as indoor MUs. Indoor macrocell interferers are denoted as dominant macrocell interferers due to the fact that the interference caused by these users at the FBSs will be higher then the outdoor macrocell interference, which may cause a significant degrade in the FU performance. Within the macrocell area, we assume that there are buildings with multiple apartment/offices and multiple femtocells, which consists of the indoor macrocell and femtocell users. Each of these buildings denotes a femtocell cluster. The scenario is depicted in Fig. 8. Each femtocell cluster, i.e., indoor block is approximated by a circular area with a radius of $80 \mathrm{~m}$. The yellow circles represent the femtocell coverage areas. As can be seen from Fig. 8, a single femtocell cluster is present in each orthogonal dimension group. In this second set of simulations, we consider the coordinated MMSE approach only given its superior performance to the coordinated zeroforcing approach.

Fig. 9 represents such a system with 70 MUs, with 10 macrocell interferers in each femtocell cluster consisting of 3 FBSs with 3 FUs in each femtocell. An indoor MU, which causes very high interference to the FUs, is called a dominant macrocell interferer. The outdoor macrocell interferers/pedestrians are called the weak macrocell interferers. The figure shows the average BER of the FUs in the system versus the number of dominant macrocell interferers per group, for a fixed total of 70 MUs. For Figs. 10-12, all MUs are considered dominant interferers. In Fig. 10, we plot the BER of the FUs versus the number of MUs per cluster. In Fig. 11, the average BER of the FUs versus FU transmit power is plotted for various number of MUs per cluster and is compared to the baseline scheme without IA. The maximum received SINR

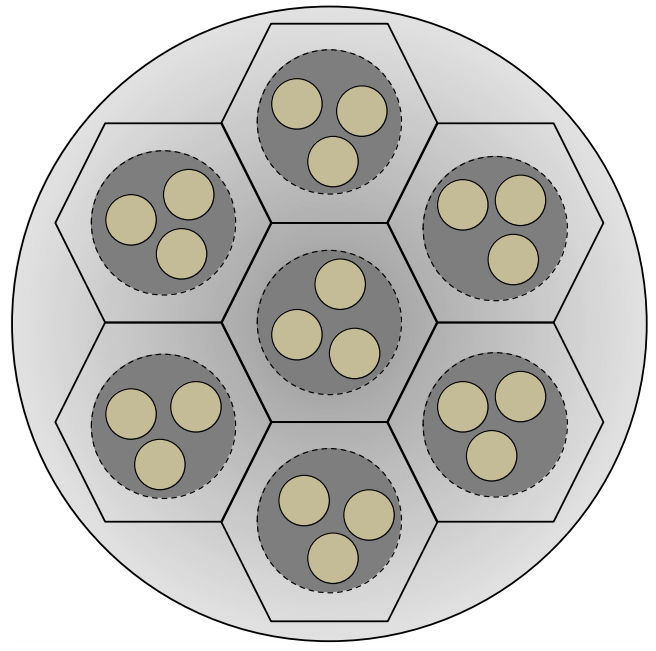

Fig. 8. Hexagonal model demonstrating the femtocell cluster formation and indoor/outdoor areas.

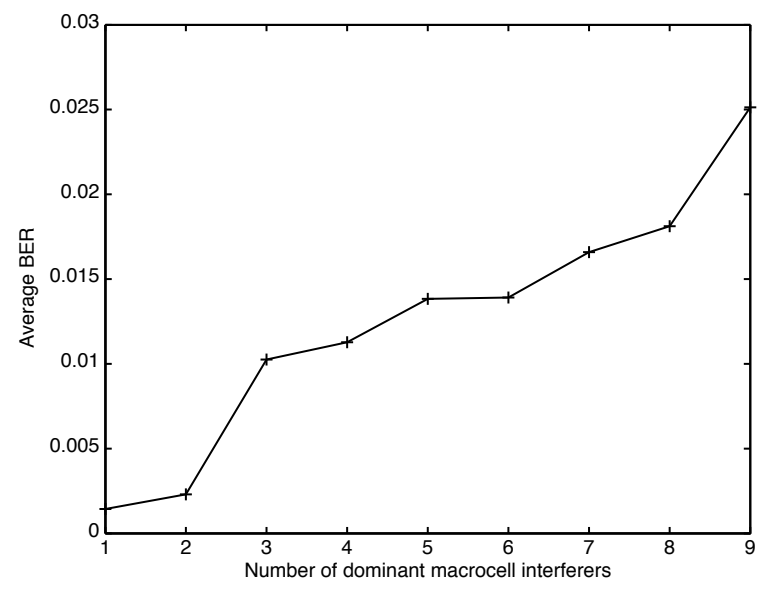

Fig. 9. Average BER for femtocell users for a fixed number of macrocell users.

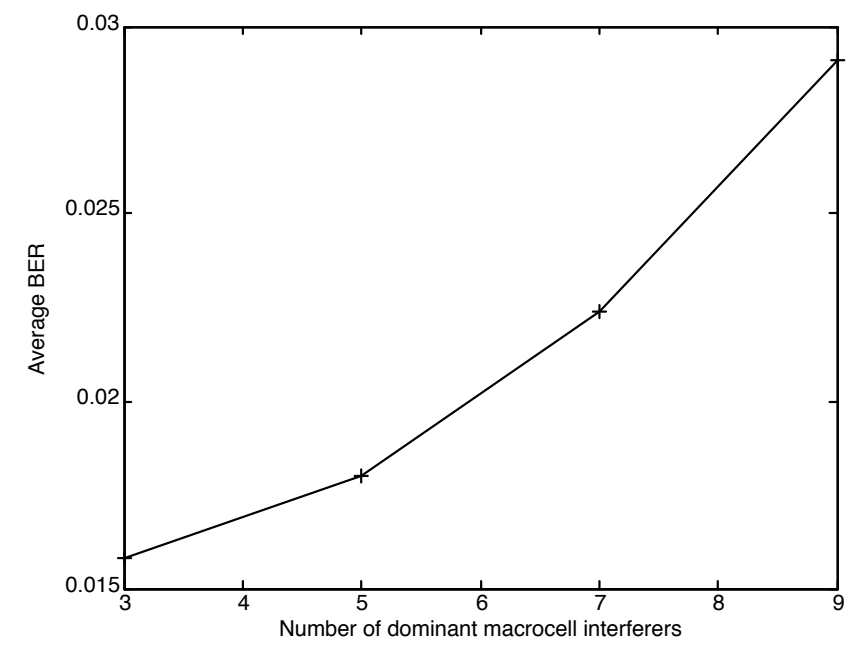

Fig. 10. Average BER for femtocell users for a variable number of macrocell users. 


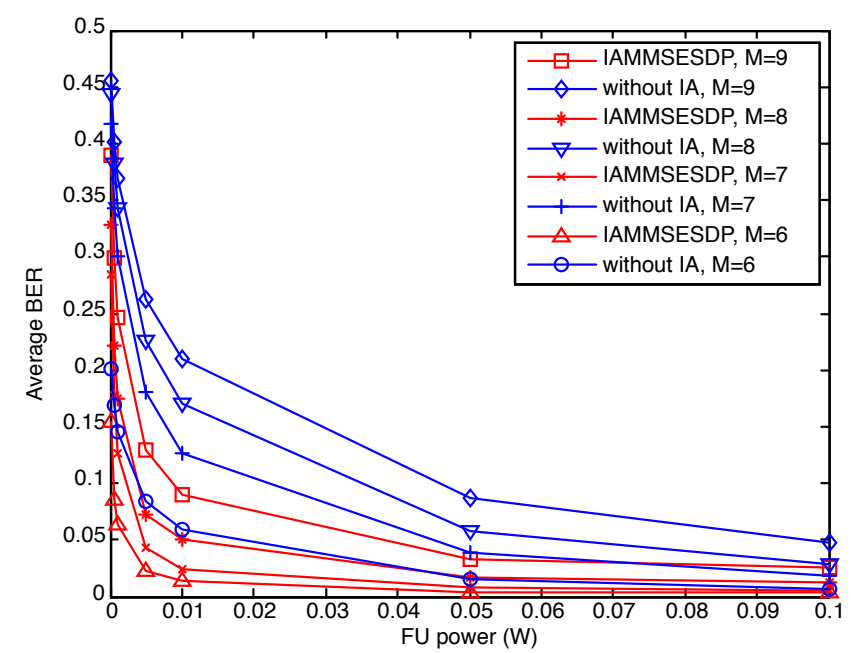

Fig. 11. Average BER of the femtocell users with and without SDP-IAMMSE algorithm.

that can be achieved as the number of interfering MUs per cluster increases is shown in Fig. 12. The average BER of the FUs for the coordinated MMSE approach is compared to zero-forcing interference cancellation in Fig. 13 subject to the number of receive antennas at the FBS, from which we observe the greater advantage of using IA in resource limited scenarios with smaller antenna numbers. Overall, the numerical results demonstrate that the performance of the FUs in terms of average BER is significantly better when compared to the case when the interfering MUs only consider their own performance and minimize the sum MSE at the MBS. The feasibility of the minimum SINR constraints is a main limitation in this system: as the minimum SINR constraints of MUs are increased, the maximum number of MUs that can be aligned decreases.

\section{CONCLUSION}

We have studied interference management for a two-tier network with femtocells deployed within a macrocell. In particular, we have opted for a design that mitigates interference caused by the MUs at multiple femtocell uplinks by using IA. Since, in this coexisting two-tiered network, interfering MUs need to have their continued connectivity as well, we have proposed an IA approach with individual SINR constraints, for which end we utilize successive SDP approximations to a quadratically constraint quadratic problem (QCQP). The algorithm is applied to mitigate uplink macrocell interference in femtocell networks and numerical results are provided to demonstrate the effectiveness of the proposed design. Future directions include system level applications of the proposed algorithms for analyzing their performance under standardcompliant scenarios, IA applications in tiered networks with reduced complexity, the impact of limited/noisy CSI, as well as including the resource constraints of the backhaul in the interference management problem.

\section{Appendix: Extension to Multiple Data Streams}

Assume that the precoder of $\mathrm{MU} j$ is given as $\mathbf{W}_{j}^{o}=$ $\left[\mathbf{w}_{j 1}^{o} \ldots, \mathbf{w}_{j d}^{o}\right]$ where $d$ is the number of bit streams transmitted. Then conditions for IA at $F$ FBSs can be given as:

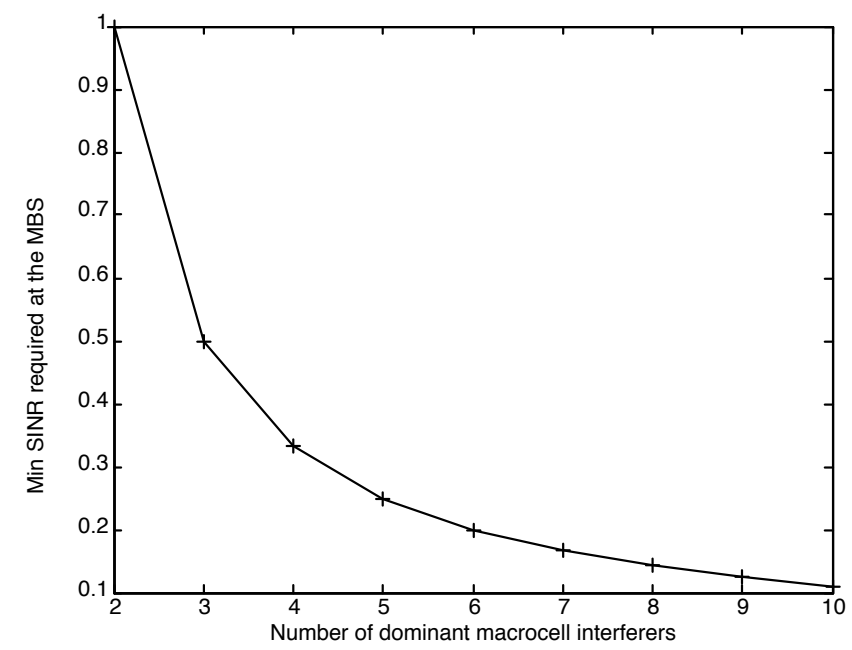

Fig. 12. Number of MUs that can be aligned subject to minimum SINR requirement at the MBS with the SDP-IA-MMSE algorithm.

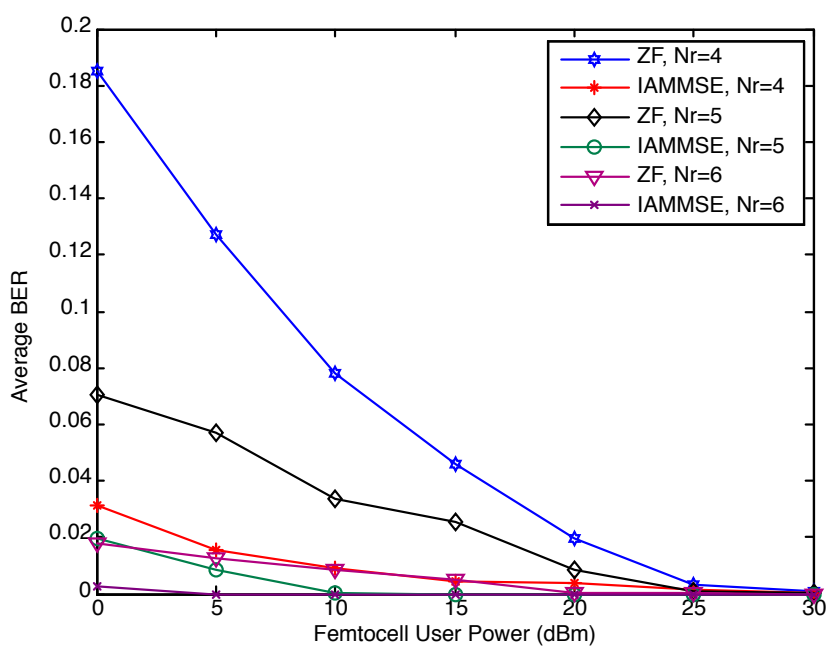

Fig. 13. Average BER of the FUs for zero-forcing interference cancellation vs. SDP-IA-MMSE.

$$
\mathbf{H}_{k 1}^{o} \mathbf{W}_{1}^{o} \prec \mathbf{H}_{k 2}^{o} \mathbf{W}_{2}^{o} \prec \cdots \prec \mathbf{H}_{k M}^{o} \mathbf{W}_{M}^{o}, k=1, \ldots, F
$$

where $\mathbf{X} \prec \mathbf{Y}$ shows that the column space of $\mathbf{Y}$ spans that of $\mathbf{X}$. Equivalently, the conditions in (33) can be expressed for the FBSs $k=1, \ldots, F$ as a system of linear equalities as:

$$
\mathbf{H}_{k 1}^{o} \mathbf{w}_{1 i}^{o}=\sum_{j=1}^{d} \alpha_{k m}^{i j} \mathbf{H}_{k m}^{o} \mathbf{w}_{m j}^{o}, \quad \forall m \in\{2, \ldots, M\}
$$

where $\alpha_{k m}^{i j}$ is a scalar coefficient as in the one-dimensional case. The linear system of equations can be represented for $k=1, \ldots, F$ in vector form as follows:

$$
\left(\mathbf{I}_{(d \times d)} \otimes \mathbf{H}_{k 1}^{o}\right) \mathbf{w}_{1}^{o}=\left(\mathbf{A}_{k m} \otimes \mathbf{H}_{k m}^{o}\right) \mathbf{w}_{m}^{o}, \quad m=2, \ldots, M
$$

where $\mathbf{w}_{m}^{o}$ denotes the vectorized form of the precoder matrix $\mathbf{W}_{m}^{o}$, in other words, $\mathbf{w}_{m}^{o}=\left[\left(\mathbf{w}_{j 1}^{o}\right)^{T} \ldots,\left(\mathbf{w}_{j d}^{o}\right)^{T}\right]^{T} . \mathbf{A}_{k m}$ denotes the $(d \times d)$ coefficient matrix formed by assigning $\alpha_{k m}^{i j}$ as the element at row $i$ and column $j$. Then (6) can be redefined for the multi-dimensional case as:

$$
\mathbf{H w}=\mathbf{0}
$$


where

$$
\begin{aligned}
& \mathbf{w}=\left[\begin{array}{llllll}
\mathbf{w}_{1}^{o T} & \mathbf{w}_{2}^{o T} & \mathbf{w}_{3}^{o T} & \ldots & \mathbf{w}_{M-1}^{o}{ }^{T} & \mathbf{w}_{M}^{o T}
\end{array}\right]^{T} \\
& \mathbf{H}=\left[\begin{array}{cccc}
\mathbf{I}_{(d \times d)} \otimes \mathbf{H}_{11}^{o} & -\mathbf{A}_{12} \otimes \mathbf{H}_{12}^{o} & \ldots & \mathbf{0} \\
\vdots & \vdots & \ddots & \vdots \\
\mathbf{I}_{(d \times d)} \otimes \mathbf{H}_{11}^{o} & \mathbf{0} & \ldots & -\mathbf{A}_{1 M} \otimes \mathbf{H}_{1 M}^{o} \\
\vdots & \vdots & \ddots & \vdots \\
\mathbf{I}_{(d \times d)} \otimes \mathbf{H}_{F 1}^{o} & -\mathbf{A}_{F 2} \otimes \mathbf{H}_{F 2}^{o} & \ldots & \mathbf{0} \\
\vdots & \vdots & \ddots & \vdots \\
\mathbf{I}_{(d \times d)} \otimes \mathbf{H}_{F 1}^{o} & \mathbf{0} & \ldots & -\mathbf{A}_{F M} \otimes \mathbf{H}_{F M}^{o}
\end{array}\right]
\end{aligned}
$$

Thus, the objective function of the SDP problem for multiple data streams is formulated. We now turn to the derivation of the new SINR constraints with matrix precoders. The SINR constraint for $\mathrm{MU} i$ with precoder $\mathbf{W}_{i}^{o}$ is given as:

$$
S I N R_{i}=\frac{\operatorname{tr}\left(\left(\mathbf{W}_{i}^{o}\right)^{H}\left(\mathbf{H}_{o i}^{o}\right)^{H} \mathbf{H}_{o i}^{o} \mathbf{W}_{i}^{o}\right)}{\sum_{\substack{n=1 \\ n \neq i}}^{M} \operatorname{tr}\left(\left(\mathbf{W}_{n}^{o}\right)^{H}\left(\mathbf{H}_{o n}^{o}\right)^{H} \mathbf{H}_{o n}^{o} \mathbf{W}_{n}^{o}\right)+\beta+\sigma^{2}}
$$

where $\mathbf{W}_{n}^{o}$ is the precoder of MU $n$ and

$$
\beta=\sum_{f=1}^{F} \sum_{u=1}^{U_{f}} \operatorname{tr}\left(\left(\mathbf{W}_{u}^{f}\right)^{H}\left(\mathbf{H}_{o u}^{f}\right)^{H} \mathbf{H}_{o u}^{f} \mathbf{W}_{u}^{f}\right)
$$

where $\mathbf{W}_{u}^{f}$ denotes the precoder for FU $u$ and $\beta$ represents the femtocell interference at the MBS. We start by re-writing the term representing the received signal strength for MU $u$ :

$$
\begin{aligned}
\operatorname{tr}\left(\left(\mathbf{W}_{n}^{o}\right)^{H}\left(\mathbf{H}_{o n}^{o}\right)^{H} \mathbf{H}_{o n}^{o} \mathbf{W}_{n}^{o}\right) \\
\quad=\sum_{j=1}^{d} \operatorname{tr}\left(\left(\mathbf{w}_{n j}^{o}\right)^{H}\left(\mathbf{H}_{o n}^{o}\right)^{H} \mathbf{H}_{o n}^{o} \mathbf{w}_{n j}^{o}\right) \\
=\left(\mathbf{w}_{n}^{o}\right)^{H}\left(\mathbf{I}_{(d \times d)} \otimes\left(\left(\mathbf{H}_{o n}^{o}\right)^{H} \mathbf{H}_{o n}^{o}\right)\right) \mathbf{w}_{n}^{o} \\
\quad=\mathbf{w}^{H}\left(\mathbf{e}_{n} \otimes \mathbf{I}_{(d \times d)} \otimes\left(\left(\mathbf{H}_{o n}^{o}\right)^{H} \mathbf{H}_{o n}^{o}\right)\right) \mathbf{w}
\end{aligned}
$$

The SDP problem for users with multiple data streams can now be formulated as:

$$
\begin{array}{ll}
\underset{\mathbf{w}_{1}^{o}, \ldots, \mathbf{w}_{M}^{o}}{\operatorname{minimize}} & \operatorname{tr}(\mathbf{R W}) \\
\text { subject to } & \operatorname{tr}\left(\left(\mathbf{R}_{o i}-\gamma_{i} \sum_{n \neq i} \mathbf{R}_{o n}\right) \mathbf{W}\right) \geq \gamma_{i}\left(\sigma^{2}+\beta\right) \\
& \operatorname{tr}\left(\left(\operatorname{diag}\left(\mathbf{e}_{i}\right) \otimes \mathbf{I}_{\left(N_{t} d \times N_{t} d\right)}\right) \mathbf{W}\right) \leq \mathrm{P}_{i}^{o} \\
& \mathbf{W} \succeq 0, \quad i=1, \ldots, M
\end{array}
$$

where $\mathbf{R}=\mathbf{H}^{H} \mathbf{H}, \mathbf{W}=\mathbf{w w}^{H}$ and $\mathbf{R}_{\text {on }}=\operatorname{diag}\left(\mathbf{e}_{n}\right) \otimes$ $\mathbf{I}_{(d \times d)} \otimes\left(\left(\mathbf{H}_{o n}^{o}\right)^{H} \mathbf{H}_{o n}^{o}\right), \forall n$. Interference alignment with SINR constraints for multiple bit streams can therefore be formulated as an SDP problem with the appropriate modifications. A detailed discussion on the properties of IA with least squares for multiple bit streams is available in [34].

\section{REFERENCES}

[1] V. Chandrasekhar, J. Andrews, and A. Gatherer, "Femtocell networks: a survey," IEEE Commun. Mag., vol. 46, no. 9, pp. 59-67, Sept. 2008.

[2] Picochip, "The case for home base stations," White Paper, Apr. 2007.

[3] S. Verdu, Multiuser Detection. Cambridge University Press, 1998.
[4] R. Yates, "A framework for uplink power control in cellular radio systems," IEEE J. Sel. Areas Commun., vol. 13, no. 7, pp. 1341-1348, Sept. 1995.

[5] C. Rose, S. Ulukus, and R. Yates, "Wireless systems and interference avoidance," IEEE Trans. Wireless Commun., vol. 1, no. 3, pp. 415-428, July 2002.

[6] W. M. Jang, B. R. Vojcic, and R. L. Pickholtz, "Joint transmitter-receiver optimization in synchronous multiuser communications over multipath channels," IEEE Trans. Commun., vol. 46, pp. 269-278, Feb. 1998.

[7] H. Jo, C. Mun, J. Moon, and J. Yook, "Interference mitigation using uplink power control for two-tier femtocell networks," IEEE Trans. Wireless Commun., vol. 8, no. 10, pp. 4906-4910, Oct. 2009.

[8] V. Chandrasekhar and J. G. Andrews, "Uplink capacity and interference avoidance for two-tier femtocell networks," IEEE Trans. Wireless Commun., vol. 8, no. 7, pp. 3498-3509, July 2009.

[9] V. R. Cadambe and S. A. Jafar, "Interference alignment and degrees of freedom region for the $\mathrm{K}$ user interference channel," IEEE Trans. Inf. Theory, vol. 54, no. 8, Aug. 2008.

[10] K. S. Gomadam, V. R. Cadambe, and S. A. Jafar, "A distributed numerical approach to interference alignment and applications to wireless interference networks," IEEE Trans. Inf. Theory, vol. 57, no. 6, pp. 3309-3322, June 2011.

[11] D. A. Schmidt, C. Shi, R. A. Berry, M. L. Honig, and W. Utschick, "Minimum mean squared error interference alignment," in Proc. 2009 Asilomar Conf. Signals, Syst., Comput., pp. 1106-1110.

[12] S. W. Peters and R. W Heath, Jr., "Cooperative algorithms for MIMO interference channels," IEEE Trans. Veh. Technol., vol. 60, no. 1, pp. 206-218, Jan. 2011.

[13] S. Ulukus and A. Yener, "Iterative transmitter and receiver optimization for CDMA networks," IEEE Trans. Wireless Commun., vol. 3, no. 6, pp. 1974-1979, Nov. 2004.

[14] H. Lv, T. Liu, X. Hou, and C. Yang, "Adaptive interference alignment for femtocell networks," in Proc. 2010 IEEE Int. Conf. Signal Process.

[15] F. Pantisano, M. Bennis, W. Saad, and M. Debbah, "Cooperative interference alignment in femtocell networks," in Proc. 2011 IEEE Global Telecommun. Conf.

[16] W. Shin, W. Noh, K. Jang, and H. Choi, "Hierarchical interference alignment for downlink heterogeneous networks," IEEE Trans. Wireless Commun., vol. 11, no. 12, pp. 4549-4559, Dec. 2012.

[17] T. Weber, I. Maniatis, A. Sklavos, and Y. Liu, "Joint transmission and detection integrated network (JOINT), a generic proposal for beyond 3G systems," in Proc. 2002 Int. Conf. Telecommun., vol. 3, pp. 479-483.

[18] S. Shamai, O. Somekh, O. Simeone, A. Sanderovich, B. M. Zaidel, and H. V. Poor, "Cooperative multi-cell networks: impact of limited-capacity backhaul and inter-users links," in Proc. 2007 Int. Symp. Inf. Theory.

[19] O. Somekh, O. Simeone, A. Sanderovich, B. M. Zaidel, and S. Shamai, "On the impact of limited-capacity backhaul and inter-users links in cooperative multicell networks," in Proc. 2008 Conf. Inf. Sciences Syst., pp. $776-780$.

[20] S. Chatzinotas and B. Ottersten, "Interference alignment for clustered multicell joint decoding," in Proc. 2011 IEEE Wireless Commun. Netw. Conf., pp. 1966-1971.

[21] H. Yu and Y. Sung, "Least squares approach to joint beam design for interference alignment in multiuser multi-input multi-output interference channels," IEEE Trans. Signal Process., vol. 58, no. 9, Sept. 2010.

[22] M. Kojima and L. Tuncel, "On the finite convergence of successive SDP relaxation methods," European J. Operations Research, 1999.

[23] S. Boyd and L. Vandenberghe, Convex Optimization. Cambridge University Press, 2004.

[24] D. Peaucelle, D. Henrion, Y. Labit, and K. Taitz, Users Guide for SeDuMi Interface 1.04. LAAS-CNRS, 2002.

[25] Z. Luo, W. Ma, A. M. So, Y. Ye, and S. Zhang, "Semidefinite relaxation of quadratic optimization problems," IEEE Signal Process. Mag., vol. 27, no. 3, pp. 20-34, May 2010.

[26] C. M. Yetis, T. Gou, S. A. Jafar, and A. H. Kayran, "On feasibility of interference alignment in MIMO interference networks," IEEE Trans. Signal Process., vol. 58, no. 9, pp. 4771-4782, Sept. 2010.

[27] G. Bresler, D. Cartwright, and D. Tse, "Settling the feasibility of interference alignment for the MIMO interference channel: the symmetric square case," ITW, Brazil, 2011.

[28] S. Serbetli and A. Yener, "Transceiver optimization for multiuser MIMO systems," IEEE Trans. Signal Process., vol. 52, no. 1, pp. 214-226, Jan. 2004.

[29] B. Guler and A. Yener, "Interference alignment for cooperative MIMO femtocell networks," in Proc. 2011 IEEE Global Telecommun. Conf., pp. 1-5. 
[30] X. Sun, L. J. Cimini, L. J. Greenstein, D. S. Chant, and J. Kruys, "Coordinated zero-forcing beamforming in multipoint MIMO networks for backhaul applications," in Proc. 2009 IEEE Military Commun. Conf.

[31] Z. Pan, K.-K. Wong, and T.-S. Ng, "Generalized multiuser orthogonal space-division multiplexing," IEEE Trans. Wireless Commun., vol. 3, no. 6, pp. 1969-1973, Nov. 2004.

[32] Q. H. Spencer, A. L. Swindlehurst, and M. Haardt, "Zero-forcing methods for downlink spatial multiplexing in multiuser MIMO channels," IEEE Trans. Signal Process., vol. 52, no. 2, pp. 461-471, Feb. 2004.

[33] ITU-R Recommendation M.1225: "Guidelines for evaluation of radio transmission technologies for IMT-2000," Feb. 1997.

[34] H. Yu, Y. Sung, H. Kim, and Y. H. Lee, "Beam tracking for interference alignment in slowly-fading MIMO interference channels: a perturbations approach under a linear framework," IEEE Trans. Signal Process., vol. 60, no. 4, pp. 1910-1926, Apr. 2012.

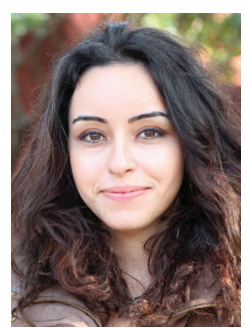

Basak Guler (S'13) received her B.Sc. degree in electrical and electronics engineering from Middle East Technical University (METU), Ankara, Turkey in 2009 and her M.Sc. degree in electrical engineering from Wireless Communications and Networking Laboratory, Pennsylvania State University, University Park, PA, in 2012. She is currently pursuing the Ph.D. degree with the Wireless Communications and Networking Laboratory, Pennsylvania State University. Her research interests include interference management in heterogeneous wireless networks, femtocell-macrocell communication systems, semantic source coding, data compression and semantic information theory.

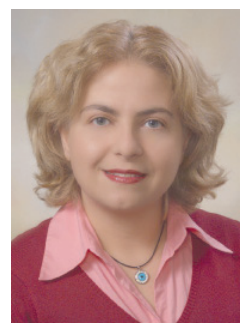

Aylin Yener (S'91-M'00-SM'13) received the B.Sc. degree in electrical and electronics engineering, and the B.Sc. degree in physics, from Bogazici University, Istanbul, Turkey; and the M.S. and Ph.D. degrees in electrical and computer engineering from Wireless Information Network Laboratory (WINLAB), Rutgers University, New Brunswick, NJ. Commencing fall 2000, for three semesters, she was a P.C. Rossin Assistant Professor at the Electrical Engineering and Computer Science Department, Lehigh University, PA. In 2002, she joined the faculty of The Pennsylvania State University, University Park, PA, where she was an Assistant Professor, then Associate Professor, and is currently Professor of Electrical Engineering since 2010. During the academic year 2008-2009, she was a Visiting Associate Professor with the Department of Electrical Engineering, Stanford University, CA. Her research interests are in information theory, communication theory and network science, with recent emphasis on green communications and information security. She received the NSF CAREER award in 2003.

Dr. Yener previously served as a technical program chair or co-chair for various conferences for the IEEE Communications Society, as an associate editor for the IEEE TRANSACTIONS ON COMMUNICATIONS, as an associate editor and an editorial advisory board member for the IEEE TRANSACTIONS ON WIRELESS COMMUNICATIONS. She served as the student committee chair for the IEEE Information Theory Society 2007-2011, and was the co-founder of the Annual School of Information Theory in North America co-organizing the school in 2008, 2009 and 2010. Dr. Yener currently serves on the board of governors of the IEEE Information Theory Society as its treasurer. 